$$
\left(c\left[g^{\prime}(z)\right]^{*}+z g^{\prime}(z)\right)-\lambda z g^{\prime}(z)=m g(z)-\lambda z g^{\prime}(z) .
$$

Then the lemma gives $p \leqq p_{1}$. This completes the proof of the theorem.

\title{
REFERENCES
}

1. G. Ancochea, Sur les polynomes dont les zeros son symetriques par rapport d une circonference, C. R. Acad. Sci. Paris vol. 221 (1945) pp. 13-15.

2. F. F. Bonsall and M. Marden, Zeros of self-inversive polynomials, Proc. Amer. Math. Soc. vol. 3 (1952) pp. 471-475.

3. A. Cohn, Über die Anrahl der Wurzeln einer algebraischen Gleichung in einer Kreise, Math. Zeit. vol. 14 (1922) pp. 110-148.

UNIVERSITY OF MADRID

\section{MEASURE EXTENSIONS AND THE MARTINGALE CONVERGENCE THEOREM ${ }^{1}$}

\section{SHU-TEH CHEN MOY}

1. Introduction. In $1940 \mathrm{~J}$. L. Doob proved the following martingale convergence theorem $[3] .^{2}$

Let $\left\{x_{n}, \mathcal{F}_{n}, n \geqq 1\right\}$ be a martingale with $^{3}$

$$
\sup \left\{E\left[\left|x_{n}\right|\right]: n \geqq 1\right\}<\infty \text {. }
$$

Then $\left\{x_{n}\right\}$ converges with probability 1 to a random variable $x_{\infty}$ of finite expectation.

In 1946 E. S. Andersen and B. Jessen proved some limit theorems on derivatives of set functions [1]. One of the theorems is closely related to the martingale convergence theorem and is stated below.

Let $\mathcal{F}_{1} \subset \mathfrak{F}_{2} \subset \cdots \subset \mathcal{F}_{n} \subset \cdots$ be a nondecreasing sequence of Borel fields of subsets of a nonempty set $\Omega$. Let $P$ be a probability measure defined on the smallest Borel field $\mathcal{F}_{\infty}$ containing all the $\mathcal{F}_{n}$ 's. Let $\phi$ be a bounded, countably additive set function defined on $\mathcal{F}_{\infty}$. Let $P_{n}, \phi_{n}$ be the contractions of $P, \phi$ to $\mathcal{F}_{n}$ respectively and suppose that each $\phi_{n}$ is absolutely continuous with respect to $P_{n}$. Let $x_{n}$ be the derivative of $\phi_{n}$ relative to $P_{n}$. Then $\left\{x_{n}\right\}$ converges, except on a set of $P$ measure 0 , to

Presented to the Society, April 25, 1953; received by the editors April 15, 1953.

1 This work was done while the author was Emmy Noether Fellow of Bryn Mawr College. It is based on a portion of the doctoral thesis submitted to the University of Michigan. The thesis was written under the supervision of Professor J. L. Doob of the University of Illinois.

2 Numbers in brackets refer to the bibliography.

${ }^{3}$ For the definition and properties of a martingale see [2, Chap 7]. 
the derivative of the $P$-continuous part of $\phi$ relative to $P$.

The above theorem will be designated as the A-J theorem throughout this note.

Doob has pointed out that the $x_{n}$ 's and $\mathcal{F}_{n}$ 's in the A-J Theorem form a martingale. In his discussion of the relation between his martingale convergence theorem and the A-J theorem [2, appendix, pp. 630-632] the following three conditions concerning a martingale $\left\{x_{n}, \mathcal{F}_{n}, n \geqq 1\right\}$ are studied.

1. $x_{n}$ 's are uniformly integrable.

2. There is a countably additive bounded set function $\phi$, defined on the smallest Borel field $\mathcal{F}_{\infty}$ containing all the $\mathcal{F}_{n}$ 's, of which the contraction $\phi_{n}$ to $\mathcal{F}_{n}$ is absolutely continuous with respect to the contraction $P_{n}$ of $P$ to $\mathcal{F}_{n}$ and for which $x_{n}$ is the derivative of $\phi_{n}$ relative to $P_{n}$ for every $n$.

3. Sup $\left\{E\left[\left|x_{n}\right|\right]: n \geqq 1\right\}<\infty$.

He showed that 1 implies 2 and 2 implies 3 ; and the condition 2 together with the condition that $\phi$ be absolutely continuous with respect to $P$ on $\mathcal{F}_{\infty}$ is equivalent to 1 . He then demonstrated that 3 is actually weaker than 2 by exhibiting an example of a martingale which satisfies 3 but not 2 . Thus he indicated that his martingale convergence theorem is more general than the A-J theorem as far as the convergence part is concerned. In this note I shall prove that if the basic space $\Omega$ on which the random variables $x_{n}$ are defined is the space of real sequences $\xi=\left\{\xi_{n}\right\}$ and $\mathcal{F}_{n}$ is the smallest Borel field containing the sets of the form $\left\{\left\{\xi_{n}\right\}: \xi_{1} \leqq \alpha_{1}, \cdots, \xi_{n} \leqq \alpha_{n}\right\}$ with $\alpha_{1}, \cdots, \alpha_{n}$ being any $n$ real numbers, then 2 and 3 are equivalent. This special case is of interest because by the representation theory [2, pp. 12-15], for any martingale there is one of this type which shares most of the relevant properties of the original martingale including the convergence property. More precisely, for any martingale $\left\{x_{n}, \mathcal{F}_{n}, n \geqq 1\right\}$ where $x_{n}$ 's are defined on $\Omega$ with elements $\omega$ and probability measure $P$, there is a mapping $T$ on $\Omega$ into the space of sequences:

$$
T(\omega)=\left\{x_{1}(\omega), x_{2}(\omega), \cdots, x_{n}(\omega), \cdots\right\} .
$$

Let $\mathcal{F}_{n}^{\prime}$ be the Borel field of sets in the sequence space generated by the collection of sets of the form

$$
\left\{\xi: \xi_{1} \leqq \alpha_{1}, \xi_{2} \leqq \alpha_{2}, \cdots, \xi_{n} \leqq \alpha_{n}\right\}
$$

where $\alpha_{1}, \alpha_{2}, \cdots, \alpha_{n}$ are $n$ real numbers. Let $\mathcal{F}^{\prime}$ be the smallest Borel field containing every $\mathcal{F}_{n}^{\prime}$ and $P^{\prime}$ be the probability measure defined on $\mathcal{F}^{\prime}$ by 


$$
P^{\prime}\left(\Lambda^{\prime}\right)=P\left(T^{-1}\left(\Lambda^{\prime}\right)\right) .
$$

If $x_{n}^{\prime}$ is the $n$th coordinate variable on $\Omega^{\prime}$, i.e., $x_{n}^{\prime}$ is defined by

$$
x_{n}^{\prime}(\xi)=\xi_{n},
$$

then $\left\{x_{n}^{\prime}, \mathcal{F}_{n}^{\prime}, n \geqq 1\right\}$ is a martingale under the probability measure $P^{\prime}$ and $\left\{x_{n}^{\prime}\right\}$ converges with probability 1 if and only if $\left\{x_{n}\right\}$ converges with probability 1 . Hence the martingale convergence theorem can be deduced from the A-J theorem by applying the theorem which is to be proved.

2. The measure extension theorem. We shall consider a more general case of a martingale with the index set to be any subset of the real line. Let $T$ be a set of real numbers. Let $\Omega$ be the totality of real-valued functions $\xi=\xi(t)$ defined on $T$.

$\mathcal{F}_{t}$ is the Borel field generated by the collection of sets of the form

$$
[\xi: \xi(s) \leqq \alpha]
$$

with $s \leqq t$ and $\alpha$ an arbitrary real number. $\mathcal{F}_{\infty}$ is the smallest Borel field containing all $\mathcal{F}_{i}$ 's. $P$ is a probability measure on $\mathcal{F}_{\infty}$. Let $\left\{x_{t}, \mathcal{F}_{t}, t \in T\right\}$ be a martingale under this probability measure and $\phi_{t}$ be a set function defined on $\mathcal{F}_{t}$ by

$$
\phi_{t}(\Lambda)=\int_{\Lambda} x_{t} d P
$$

Then $\phi_{t}$ is bounded, countably additive, and absolutely continuous with respect to $P_{t}$, the contraction of $P$ to $\mathcal{F}_{t}$. The derivative of $\phi_{t}$ relative to $P_{t}$ is then $x_{t}$. Furthermore, each $\phi_{t}$ is an extension of $\phi_{t}$ if $s \leqq t$. Let $\phi$ be defined on $U_{t \in T} \mathcal{F}_{t}$ by

$$
\phi(\Lambda)=\phi_{t}(\Lambda)
$$

Notice that $U_{t \in T} \mathcal{F}_{t}$ is a field of subsets of $\Omega$ and $\phi$ is a finite, realvalued, finitely additive set function on it.

THEOREM. $\phi$ can be extended to be a countably additive set function on $\mathcal{F}_{\infty}$ if and only if $\sup \left\{E\left[\left|x_{t}\right|\right]: t \in T\right\}<\infty$. The extension is then bounded.

Before proving the above theorem we shall discuss some measure preliminaries.

For a finite real-valued finitely additive set function $\phi$ defined on a field $\mathcal{A}$ of subsets of a set $\Omega$ the positive part $\phi^{+}$and the negative part $\phi^{-}$of $\phi$ are defined by the following. 


$$
\begin{aligned}
& \phi^{+}(A)=\sup [\phi(B): B \subset A, B \in \mathcal{A}], \\
& \phi^{-}(A)=-\inf [\phi(B): B \subset A, B \in \mathcal{A}] .
\end{aligned}
$$

Then $\phi^{+}, \phi^{-}$are non-negative, finitely additive set functions on $\mathcal{A}$ and

$$
\phi(A)=\phi^{+}(A)-\phi^{-}(A)
$$

if either $\phi^{+}(A)$ or $\phi^{-}(A)$ is finite. Furthermore, if $\phi$ is countably additive, then $\phi^{+}$and $\phi^{-}$are also [4, pp. 21-22]. The following lemma concerns a necessary and sufficient condition for the existence of a countably additive extension of $\phi$ to the smallest Borel field $\mathcal{F}$ containing $\mathcal{A}$.

LEMMA 1. In order that there exists a countably additive extension of $\phi$ to the smallest Borel field $\mathcal{F}$ containing $\mathcal{A}$, it is necessary and sufficient that the following two conditions be satisfied.

a. $\phi$ is countably additive on $A$.

b. $\phi$ is bounded on $A$, i.e., there is a non-negative number $K$ for which $|\phi(A)| \leqq K$ for all $A \in \mathcal{A}$.

Proof. 1. Necessity. a is obvious. For b, let $\Phi$ be a countably additive extension of $\phi$ on $\mathcal{F}$. Then $\Phi$ cannot take on both values $+\infty$ and $-\infty$. Let $\Phi^{+}$and $\bar{\phi}^{-}$be the positive part and the negative part of $\phi$ respectively, then $\bar{\phi}=\Phi^{+}-\bar{\phi}^{-}$and there are two disjoint sets $C$ and $D$ in $\mathcal{F}$ with $C \cup D=\Omega$ such that

$$
\Phi^{+}(A)=\Phi(A \cap C), \quad \Phi^{-}(A)=-\Phi(A \cap D)
$$

for every set $A \in \mathcal{F}[5$, pp. 121-123]. If $\Phi$ does not take the value $-\infty$ then

$$
\bar{\phi}^{-}(\Omega)=-\bar{\phi}(D)<\infty .
$$

Hence for every set $A \in \mathcal{F}$

$$
\boldsymbol{\phi}^{-}(A) \leqq \bar{\phi}^{-}(\Omega)=-\bar{\phi}(D)<\infty,
$$

and

$$
\Phi^{+}(A) \leqq \Phi^{+}(\Omega)=\phi(C)=\Phi(\Omega)-\phi(D)<\infty
$$

as $\phi(\Omega)=\phi(\Omega) \neq \pm \infty$. Therefore both $\phi^{+}$and $\Phi^{-}$are bounded. The same conclusion would be reached if $\phi$ does not take the value $+\infty$. The boundedness of $\phi^{+}$and $\phi^{-}$follows from the inequalities:

$$
\phi^{+}(A) \leqq \Phi^{+}(A), \quad \bar{\phi}(A) \leqq \bar{\phi}^{-}(A) .
$$

Since $\phi(A)=\phi^{+}(A)-\phi^{-}(A)$ for every $A \in \mathcal{A}, \phi$ is bounded. 
2. Sufficiency. If $\phi$ satisfies both a and $b$, then $\phi^{+}$and $\phi^{-}$are finite and countably additive. Since $\phi^{+}$and $\phi^{-}$are non-negative they can be extended to be finite-valued countably additive measures $\overline{\phi^{+}}, \overline{\phi^{-}}$on $\mathcal{F}$. For every $A \in \mathcal{A}$

$$
\overline{\phi^{+}}(A)-\overline{\phi^{-}}(A)=\phi^{+}(A)-\phi^{-}(A)=\phi(A) .
$$

Therefore $\overline{\phi^{+}}-\overline{\phi^{-}}$is a countably additive extension of $\phi$ on $\mathcal{F}$.

Using the preceding lemma we can now prove the theorem.

The $\phi$ defined by (1) and (2) is a finite-valued finitely additive set function. The domain of definition of $\phi$ is a field of sets. Suppose $\phi$ can be extended to be a countably additive set function on $\mathcal{F}_{\infty}$; by the preceding lemma there is a number $K$ for which $|\phi(\Lambda)| \leqq K$ for all $\Lambda \in U_{t \in T} \mathcal{F}_{t}$. Then

$$
\begin{aligned}
E\left[\left|x_{t}\right|\right] & =\int_{\left[x_{t} \geqq 0\right]} x_{t} d P^{\prime}-\int_{\left[x_{t}<0\right]} x_{t} d P \\
& =\phi\left(\left[x_{t} \geqq 0\right]\right)-\phi\left(\left[x_{t}<0\right]\right) \leqq 2 K .
\end{aligned}
$$

Conversely, suppose $\sup \left\{E\left[\left|x_{t}\right|\right]: t \in T\right\}=L<\infty ;$ then $\phi$ is bounded for

$$
|\phi(\Lambda)|=\left|\int_{\Lambda} x_{t} d P\right| \leqq E\left[\left|x_{t}\right|\right] \leqq L
$$

if $\Lambda \in \mathcal{F}_{t}$. To show that $\phi$ is countably additive we shall do the following.

For each $t \in T$ define a non-negative, countably additive measure $\mu_{t}$ on $\mathcal{F}_{t}$ by the equation

$$
\mu_{t}(\Lambda)=\int_{\Lambda_{t}}\left|x_{t}\right| d P .
$$

If $t \leqq t_{1} \leqq t_{2} ; t, t_{1}, t_{2} \in T ; \Lambda \in \mathcal{F}_{t}$, then $\mu_{t_{1}}(\Lambda) \leqq \mu_{t_{2}}(\Lambda) \leqq L$ for

$$
\begin{aligned}
\int_{\Lambda}\left|x_{t_{1}}\right| d P & =\int_{\Delta}\left|E\left[x_{t_{2}} \mid \mathcal{F}_{t_{1}}\right]\right| d P \leqq \int_{\Delta} E\left[\left|x_{t_{2}}\right| \mid \mathcal{F}_{t_{1}}\right] d P \\
& =\int_{\Lambda}\left|x_{t_{2}}\right| d P \leqq L .
\end{aligned}
$$

Let $b$ be the maximum value of the closure of $T$ ( $b$ may be infinity). Let $t_{1} \leqq t_{2} \leqq t_{3} \leqq \cdots$ be a sequence of elements of $T$ with $t \leqq t_{n}$ for every $n$ and $\lim _{n \rightarrow \infty} t_{n}=b$. A set function $\mu_{t}^{*}$ is defined on $\mathcal{F}_{t}$ by 


$$
\mu_{t}^{*}(\Lambda)=\lim _{n \rightarrow \infty} \mu_{t_{n}}(\Lambda)
$$

It is easy to see that $\mu_{t}^{*}$ is independent of the particular sequence $\left\{t_{n}\right\}$ chosen and is additive and finite-valued. It is also countably additive and absolutely continuous with respect to $P_{t}$, because it is the finite limit of a nondecreasing sequence of countably additive measures [5]. Furthermore, if $t<t^{\prime} ; t, t^{\prime} \in T ; \Lambda \in \mathcal{F}_{t}$, then

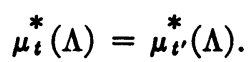

Hence a set function $\mu^{*}$ can be defined on $U_{t \in T} \mathcal{F}_{t}$ by

$$
\mu^{*}(\Lambda)=\mu_{t}^{*}(\Lambda)
$$

if $\Lambda \in \mathcal{F}_{t}$. Clearly, $\mu^{*}$ is a non-negative finite-valued additive set function on $U_{t \in T} \mathcal{F}_{t}$ and is countably additive on every $\mathcal{F}_{t}$. Kolmogorov has proved that such a set function is also countably additive on $U_{t \in r} F_{t}[0]$.

For each $\Lambda \in U_{t \in T} \mathcal{F}_{t}$,

$$
|\phi(\Lambda)| \leqq \mu^{*}(\Lambda)
$$

Hence the countable additivity of $\mu^{*}$ implies the countable additivity of $\phi$. For, let $\Lambda_{1} \supset \Lambda_{2} \supset \cdots \supset \Lambda_{n} \supset \cdots$ be any decreasing sequence of sets in $U_{t \in r} \mathcal{F}_{t}$ for which $\bigcap_{n=1}^{\infty} \Lambda_{n}=$ null set, then $\lim _{n \rightarrow \infty} \mu^{*}\left(\Lambda_{n}\right)=0$. Hence $\lim _{n \rightarrow \infty} \phi\left(\Lambda_{n}\right)=0$ and therefore $\phi$ is countably additive. Q.E.D.

\section{Bibliography}

1. E. S. Andersen and B. Jessen, Some limil theorems on integrals in an abstract set, Det Kgl. Videnskabernes Selskab, Matematisk-Fysiske Meddelelser vol. 22, No. 14, 1946.

2. J. L. Doob, Stochaslic processes, New York, Wiley.

3. - Regularity properties of certain families of chance variables, Trans. Amer. Math. Soc. vol. 47 (1940) pp. 485-486.

4. H. Hahn and A. Rosenthal, Set functions, University of New Mexico Press, 1948.

5. P. Halmos, Measure theory, Von Nostrand, 1950. 1950.

6. A. N. Kolmogorov, Foundations of the theory of probability, New York, Chelsea,

UNIVERSITY OF MICHIGAN AND

UNIVERSITY OF ILLINOIS 